\title{
Amor cultivado y amor acelerado: la relación entre los espacios y el desarrollo amoroso en Ana Karenina.
}

\section{Cultivated love and accelerated love: the relationship between spaces and love development in Ana Karenina.}

\author{
Esta obra está bajo una Licencia Creative Commons Atribución 4.0 Internacional.
} DOI: $\underline{10.32870 / \text { sincronia.axxii.n74.22b18 }}$

\author{
Laura Isabel Cortés Ladino \\ Universidad de Guadalajara \\ (MÉXICO) \\ licorteslad@gmail.com
}

Recibido: $31 / 03 / 2018$

Revisado: $24 / 04 / 2018$

Aprobado: 16/05/2018

\section{RESUMEN}

Este artículo busca dar cuenta de una relación entre los espacios físicos y el desarrollo de las relaciones amorosas en la novela Ana Karenina, del ruso Lev Tolstoi. El texto plantea que los ritmos sociales de los espacios, el urbano y el rural, son diametralmente distintos, en concordancia con las visiones ideológicas y las digresiones del autor. En relación con este aspecto también plantea una relación entre dichos ritmos y el desarrollo de las relaciones amorosas en la novela, específicamente en la de Ana y Vronsky, y la de Kitty y Levin. En este sentido explica cómo la primera transcurre de acuerdo con los ritmos urbanos, dado que se desarrolla en las ciudades; y cómo la segunda es construida en consonancia con los ritmos rurales ligados al trabajo de la agricultura.

Palabras clave: Ana Karenina. Urbano. Rural. Amor. Tolstoi.

\section{ABSTRACT}

This article's purpose is to present a relationship between the physical spaces and the development of the love relationships in the novel Ana Karenina, of Lev Tolstoi. The text states that the social rhythms of the spaces, urban and rural, are totally opposite, according with the author's ideological views and his digressions. Related with this aspect, the text also states a relationship between these rhythms and the development of the love relationships in the novel, specifically the relationships 
between Ana and Vronsky, and Kitty and Levin. In this way, the article explains how the first one elapses according to the urban rhythms, because it happens in the city; and the second one is built consistent with the rural rhythms, linked with the agriculture

Keywords: Ana Karenina. Urban. Rural. Love. Tolstoi.

El presente artículo nace de la revisión de un texto desarrollado en el marco del pregrado en Estudios Literarios de la Universidad Nacional de Colombia, y enviado como ponencia al Foro de Estudiantes de Literatura y Lingüística de la Universidad de Sonora. Es decir, este texto constituye un diálogo del texto anteriormente mencionado con los comentarios, críticas y aportes de los que ha sido objeto en ambas instituciones. El tema central es la relación entre el desarrollo de los sentimientos románticos de las parejas principales de la obra Ana Karenina, de León Tolstoi; y los espacios en los que transcurre la obra. Es decir, este artículo busca explorar un vínculo entre los escenarios y la construcción amorosa de Vronsky y Kitty, Vronsky y Ana, Stiva y Dolly y Levin y Kitty, así como acercarse a la construcción del carácter de estos personajes, también ligado al espacio. Dicha relación será entendida en función de los postulados ideológicos del propio Tolstoi. En este sentido este trabajo plantea una distinción entre los espacios urbanos: las ciudades; y los espacios rurales: el campo.

El desarrollo de Ana Karenina está determinado por las digresiones personales de Tolstoi ya que no es posible separar al Tolstoi pensador/ predicador del Tolstoi artista (Nabokov, 2010). En este sentido es importante señalar que las ideas políticas del autor valoraban favorablemente el trabajo agrario y manual del campo sobre las labores burocráticas de la ciudad, y, asimismo, a la vida sencilla del espacio rural sobre la sofisticación y las apariencias del urbano. Tolstoi vivió su vida en un dilema entre sus apetitos sensuales de la vida urbana y la vida ascética y tranquila del campo, el cual culminó con la decisión de abandonar la vida familiar y la creación estética en pos de una vida ética y sencilla (Nabokov, 2010). Esta dicotomía determinante en la vida del auto se puede apreciar en la novela a través de los ojos de Levin, el personaje que encarna al conde Lev Tolstoi en su propia obra, quien es, y se considera a sí mismo, un hombre rural. 
Levin, al principio de la novela, se muestra dividido entre su apacible vida como terrateniente en sus tierras en el campo, y la búsqueda del amor en la ciudad de Moscú. Llega a la ciudad con el objetivo de declararse a Kitty Schebartsky y pedir su mano en matrimonio. Sin embargo, Levin es un hombre de campo, incómodo antes los modales de la alta sociedad citadina, e incapaz de encajar y desenvolverse en ella. Asimismo, Levin menosprecia el trabajo urbano y burocrático, y la vida cortesana; en contraste con el trabajo de la tierra, propio del espacio rural, como evidencia en su primer encuentro con Oblonsky en la novela. Levin es un hombre con continuas luchas internas sobre la manera correcta de actuar, es decir se encuentra en un continuo proceso de reflexión sobre sí y sus acciones. Es un terrateniente, un hombre de campo, que no solo es poseedor de tierras, sino que busca trabajarlas, es decir, encuentra cierta paz y sentimiento de realización en el campo. La torpeza de Levin en la sociedad citadina y su carácter de hombre rural se hacen evidentes en la obra a partir de la mirada de la princesa Schebartsky, la mamá de Kitty, quien no lo considera digno de su hija.

No le agradaba Levin por sus opiniones violentas y raras, por su torpeza para desenvolverse en sociedad, motivada, a juicio de ella, por el orgullo. Le disgustaba la vida salvaje, según ella, que el joven llevaba en el pueblo, donde no trataba más que con animales y campesinos. (Tolstoi, 2006, p. 31).

Al igual que su madre, Kitty no considera a Levin como el pretendiente deseable para el estilo de vida citadino que desea, y lo rechaza. Por ese motivo Levin se retira dolido y harto de la ciudad, y regresa al campo, donde desarrolla una serie de reflexiones personales y sociales de carácter ético, que dan cuenta de, como mencioné anteriormente, las propias reflexiones del autor. Así, a través de la mirada de Levin, su encarnación en la obra, Tolstoi plantea esta dicotomía entre ciudad y campo.

Por su parte el personaje Kitty experimenta una transformación ligada al cambio de los espacios que habita, y es una de las trasformaciones más evidentes en la obra. Al principio de la novela Kitty es una niña citadina, mimada e ingenua, que no siente mucho interés en las personas 
que le rodean más allá de su futuro matrimonio. Esta es una Kitty inmadura, pero luego experimentará una transformación a partir de su decepción amorosa, producto del desaire de Vronsky. Después de este episodio, su familia la envía fuera de Moscú al declararla enferma. Este viaje es el inicio de su cambio. Viaja al extranjero a un balneario donde conoce, entre muchas personas, a Vareñka, quien la impresiona profundamente, y empieza a crear en ella el deseo de cambio, para dejar de ser la niña mimada del inicio de la novela. En este espacio, Kitty busca ayudar a personas enfermas y tiene un acercamiento importante a la religión, así, empieza a encontrar la tranquilidad perdida por el rechazo de Vronsky. Kitty cuestiona su entorno, su actitud hacia los demás, y en definitiva a sí misma, lo que la lleva hacia cierta actitud de serenidad y paz. "No era tan despreocupada y alegre como antes, pero estaba tranquila. El dolor que sufriera en Moscú no era ya para ella más que un recuerdo" (Tolstoi, 2006, p. 166). Después de su estadía en el balneario Kitty se traslada a Erguchevo, donde está viviendo Dolly con sus hijos, cerca del lugar donde vive Levin.

Por su parte, luego de la negativa de Kitty hacia a su propuesta de matrimonio, Levin se refugia en sus terrenos rurales, en sus relaciones con los campesinos y en el trabajo físico. Para éste el pueblo es un espacio de tranquilidad y realización, a través de la que considera la actividad más noble, el trabajo de la tierra. Así, trabaja codo a codo con los campesinos, segando las tierras para cultivar, y esto le proporciona la tranquilidad y la alegría que hacen contrapeso al desasosiego que le dejó su visita a Moscú y a Kitty. El trabajo físico rural lo absorbe y cambia sus pensamientos, empiezan a desvanecerse el dolor por el rechazo y las preocupaciones; así es totalmente feliz. Son estos cambios en cada uno, la serenidad de Kitty y la felicidad que brinda a Levin, los que luego harán posible la relación entre ambos. Estos procesos personales de cada uno, tienen un momento de encuentro y éxtasis cuando Levin, después de trabajar en el campo la observa pasar camino a la casa donde viven Dolly y sus hijos. Este encuentro fugaz le basta a Levin para que sus ideas alrededor del trabajo y la vida en el campo converjan con su amor por Kitty. En este sentido el amor de Levin y Kitty solo es posible en el espacio rural, y determinado por las dinámicas de éste. 
Levin es un personaje rural, y asimismo Kitty también se transforma en uno, al abandonar la ciudad y encontrar la paz y el amor en el campo; ambos se desarrollan lentamente y se transforman a partir de la reflexión, en un proceso lento que se puede identificar con el ritmo del trabajo agrario, propio del campo. Asimismo, en la obra existen personajes citadinos con distintas características, ritmos y desarrollos; como Oblonsky, Ana y Karenin, los Schebartsky, y Vronsky. Oblonsky es totalmente un hombre de ciudad, su preocupación principal es la satisfacción de sus deseos, y en ningún momento reflexiona sobre la dimensión moral de sus acciones, ni siquiera cuando es descubierta su infidelidad, y su matrimonio entra en crisis. Según Estanislao Zuleta (1980) esta aparición de Oblonsky tiene la función de contrastar este personaje con Levin. Zuleta señala como Oblonsky es incapaz de amar, dado su egocentrismo y su acentuado amor por sí mismo. "Esteban Arkadievich no puede amar, ni a la institutriz ni a Dolly, porque se ama demasiado a sí mismo y sólo el que se busca a sí mismo puede amar con pasión" (Zuleta, 1980, p. 32). En este sentido Oblonsky contrasta con Levin, quien no se ama a sí mismo, y es esta duda y falta de amor propio lo convierten en un personaje complejo que es capaz de amar verdadera y pasionalmente a Kitty, a quien cree no merecer.

Por su parte Vronsky también es un personaje evidentemente urbano, lo cual está en consonancia con la simpatía y atracción que siente por Oblonsky al principio de la obra. También pertenece a la capital, y se mueve en los círculos sociales urbanos con facilidad y fluidez, Vrosnky es todo lo contrario al rústico Levin, por quien incluso experimenta cierto rechazo al conocerlo, y decir a Oblonsky que cree que Kitty puede estar con alguien mejor. Nabokov (2010) evidencia esta diferencia al señalar que Vronsky actúa movido por la satisfacción de sus deseos e impulsos, sin reparar en la dimensión moral de sus actos, como evidencia su coqueteo con Kitty o incluso su relación con Ana, no existe un cambio en este aspecto; mientras que Levin se caracteriza por las reflexiones morales, su evolución espiritual y su sentimiento de deber con el mundo que lo rodea.

Liovin es muy distinto de Vronski, que es un hombre que vive solo para satisfacer sus impulsos. Antes de conocer a Ana, Vronski ha llevado una vida convencional; incluso en el 
amor, se contenta con sustituir los ideales morales por los modos y maneras de su círculo social. Pero Liovin es un hombre que siente el deber de comprender inteligentemente el mundo circundante y averiguar cuál es el puesto que dentro de él le corresponde. Por lo tanto su carácter está en constante evolución, va creciendo espiritualmente a lo largo de toda la novela (Nabokov, 2010, p. 238).

La evolución (o falta de ella) de estos personajes está ligada a los espacios físicos donde se encuentran, estos espacios configuran personajes distintos. Cuando Levin se encuentra en la ciudad actúa de manera torpe y se siente continuamente incómodo en su trato con "el gran mundo", mientras que en el trabajo práctico y manual en el campo encuentra paz y respuestas para sus continuas meditaciones. Por su parte, cuando conoce a Levin, Vronsky afirma que le aburriría vivir en el campo, y aunque luego se traslada a éste con Ana, su vida allí no se basa en el trabajo físico y práctico, como es el caso de Levin, sino en sus relaciones en sociedad y la vida cortesana, una actividad propiamente citadina. La prioridad de Levin es el trabajo, mientras que la de Vrosnky es la vida social, aspectos de su personalidad que determinaran en gran medida sus relaciones con las mujeres que aman.

Asimismo, los Karenin, Ana y su esposo, también son personajes citadinos, que viven no en Moscú, sino en San Petersburgo. Estas ciudades son espacios urbanos distintos, ya que Moscú es una ciudad más pequeña y provinciana comparada con el gran mundo de la capital, San Peterburgo (Nabokov, 2010). Ana es una mujer de ese gran mundo: es considerada por la sociedad como distinguida, respetable y sofisticada, al principio de la obra todas las personas con las que tiene contacto la admiran y aprecian. Está casada con un hombre reconocido y respetado, en el que se fundamenta una parte importante de su prestigio. A pesar de no ser completamente feliz con su vida y su matrimonio, se encuentra bien posicionada en sociedad en San Petersburgo. Es decir, es una mujer cosmopolita. Ana ha vivido una vida cómoda y monótona en la capital, pero cuando sale de ella para dirigirse a la provinciana Moscú conoce a Vronsky, y este encuentro le revela una nueva manera de ver el mundo. Su primer contacto con el amor y la pasión le permiten darse cuenta de 
que no es feliz, y se siente atrapada en aquella vida tan respetable y admirada, lo cual se hace evidente cuando al regresar ve a su marido de una manera que no había visto antes.

Hay esa famosa escena en la estación de ferrocarril de San Petersburgo, cuando Karenin acude a recibirla a su regreso de Moscú, y ella de repente se fija en el tamaño y la extraña convexidad de sus orejas, enormes y domésticas. Nunca hasta entonces se había fijado en esas orejas porque nunca le había mirado con ojos críticos; había sido para ella una de las cosas aceptadas de la vida, incluidas en su propia vida aceptada. Ahora todo ha cambiado. Su pasión por Vronski es un chorro de blanca luz bajo el cual lo que antes era su mundo parece un paisaje muerto de un planeta muerto (Nabokov, 2010, p. 95).

La relación de Ana y Vronsky tiene un ritmo totalmente distinto al de Kitty y Levin. Es apasionada, intensa y no se basa en las reflexiones de las dos personas involucradas en ella, sino en los arrebatos amorosos. Después de pasar de ser una respetable mujer citadina con un matrimonio ideal a abandonarlo todo, incluyendo a su hijo, para darle rienda suelta a su pasión; Ana se transforma. Poco a poco va perdiendo la sobriedad que la caracterizaba al inicio de la novela, y se hace parte de la intensa marea de sentimientos que ha despertado en ella su relación con Vronsky. La relación de Ana y Vronsky inicia en Moscú y se desarrolla en San Petersburgo, ocurre en los espacios sociales urbanos que son denominados como "el gran mundo", es decir, ésta es una relación propia de dos personajes urbanos y socialmente admirados por las personas de la ciudad, pero paradójicamente la relación misma trunca las relaciones de los involucrados, especialmente la de Ana con este "gran mundo". Sin embargo, no es el adulterio lo que pone a la alta sociedad en contra de Ana, sino la exhibición de la subversión. Otras mujeres, como la princesa Betsy, también han tenido relaciones extramaritales de las cuales se tiene conocimiento, pero éstas se mantuvieron bajo un pretendido secreto Era comprensible, e incluso esperable, que Vronsky que cortejara a Ana, dado que es una conducta normal e incluso atractiva entre los jóvenes de "el gran mundo", como confirma la actitud de Veselovsky hacia Kitty, frente a Levin; pero es importante que estas faltas se mantengan sutiles, y que no pasen de ser una discreción. Al contrario, Ana expone su amor por 
Vronsky públicamente, sin demostrar vergüenza o pudor, y en este sentido su crimen es no avergonzarse de lo que ha hecho, es obligar a este gran mundo a observar su desobediencia.

No es acertado afirmar que la convivencia de Levin y Kitty es completamente armónica y funcional después de su matrimonio, y que éste sea el factor que les diferencia de Ana y Vronsky. La diferencia principal entre ambas relaciones no puede ser concebida desde una visión simplista de buena y mala, no quiere decir que la relación de Levin y Kitty sea la buena, y la de Ana y Vronsky la mala, existen continuos conflictos entre los primeros, ya sea a causa de celos, o de las continuas reflexiones y dudas que atormentan constantemente a Levin. En este sentido lo que distingue a ambas relaciones no es la ausencia o presencia de conflictos, sino el ritmo de cada una. El amor entre Levin y Kitty es un amor lento y en constante construcción, mientras que el de Vronsky y Ana es una pasión inmediata y abrasadora. Levin y Kitty construyen lentamente su relación, al mismo tiempo en que se construyen a sí mismos y cambian a partir de sus reflexiones; mientras que Ana y Vronsky se entregan el uno al otro al apenas conocerse, y sus prioridades empiezan a ser sentimientos que cada uno de ellos despierta en el otro.

Por este motivo este texto afirma que los ritmos amorosos en la novela están vinculados cada uno con los espacios físicos donde transcurre la narración. La relación de Levin y Kitty es un amor cultivado, con ritmo del trabajo agrario y rural, paciente y continuo, cuyos resultados se aprecian con el tiempo. Al igual que con las cosechas, el amor de Levin y Kitty ha requerido un esfuerzo continuo para florecer, se marchita y vuelve a empezar. Por su parte el amor de Vrosnky y Ana es un amor acelerado que tiene el ritmo caótico y acelerado de la ciudad: desde la primera vez que se ven, surge entre ambos una atracción intensa que se despliega rápidamente, y los arrolla sin que puedan oponerse, y aunque ambos llegan a tener reparos sobre ésta, no pueden dejarse el uno al otro, y solo el suicidio de Ana pone fin a su situación. Así pues, Levin y Kitty trabajan y construyen su amor, mientras Ana y Vronsky son arrastrados por el suyo.

\section{Referencias:}


Nabokov, V. (2010). Curso de literatura rusa. Barcelona, España. RBA libros.

Tolstoi, L. (2006) Anna Karenina. Recuperado de: http://biblioteca.org.ar/libros/133590.pdf

Zuleta, E. (1980) La propiedad, el matrimonio y la muerte en Tolstoi. Bogotá, Colombia. Nuevaletra. 